\title{
Enhancement of Atmospheric Radiation by an Aerosol Layer
}

\author{
Diane V. Michel.angeli, Mark Alien, ${ }^{1}$ Yuk L. Yung, Run-Lie Shia, \\ DAVID CRISP, ${ }^{1}$ and JANUSZ ELUSZKIEWICZ \\ Division of Geological and Planetary Sciences, Califomia Institute of Technology, Pasadena
}

\begin{abstract}
The presence of a stratospheric haze layer may produce increases in both the actinic flux and the irradiance below this layer. Such haze layers result from the injection of aerosol-forming material into the stratosphere by volcanic eruptions. Simple heuristic arguments show that the increase in flux below the haze layer, relative to a clear sky case, is a consequence of "photon trapping." We explore the magnitude of these flux perturbations, as a function of aerosol properties and illumination conditions, with a new radiative transfer model that can accurately compute fluxes in an inhomogeneous atmosphere with nonconservative scatterers having arbitrary phase function. One calculated consequence of the El Chichon volcanic eruption is an increase in the midday surface actinic flux at $20^{\circ} \mathrm{N}$ latitude, summer, by as much as $45 \%$ at $2900 \AA$. This increase in flux in the UV-B wavelength range was caused entirely by aerosol scattering, without any reduction in the overhead ozone column.
\end{abstract}

\section{INTRODUCTION}

The actinic flux and the irradiance are two important radiometric quantities that are often confused with each other [Madronich, 1987]. The actinic flux (sometimes referred to as the average intensity) is utilized in calculating photodissociation rate constants and has two components. (1) The attenuated direct solar beam at wavelength $\lambda$ is defined by

$$
J_{0}(z, \lambda)=\pi F_{0}(\lambda) e^{-\tau(z) / \mu_{0}},
$$

where $\pi F_{0}(\lambda)$ is the solar flux per unit area perpendicular to the direction of propagation at the top of the atmosphere, $\tau(z)$ is the optical depth at altitude $z$, and $\mu_{0}=\cos \theta$ where $\theta_{0}=$ solar zenith angle. (2) The diffuse actinic flux is defined by

$$
J_{D}(z, \lambda)=\int_{4 \pi} I_{\lambda}(z, \Omega) d \Omega,
$$

where $I_{\lambda}(z, \Omega)$ is the diffuse specific intensity per solid angle $\Omega$. Finally, the total actinic flux is obtained by adding the direct and the diffuse fluxes,

$$
J(z, \lambda)=J_{0}(z, \lambda)+J_{D}(z, \lambda)
$$

as discussed by Wofsy [1978]. Rayleigh-scattered diffuse flux could be as much as $60 \%$ of the total actinic flux at wavelengths important for the photolysis of $\mathrm{ClNO}_{3}$ in the stratosphere. The diffuse flux is a significant term in the calculation of the photodissociation rate constants of other molecules including $\mathrm{NO}_{2}, \mathrm{~N}_{2} \mathrm{O}_{5}, \mathrm{HOCl}$, and $\mathrm{O}_{3}$. Accurate numerical algorithms now exist for properly calculating dissociation values in the presence of scattering [Yung, 1976; Luther and Gelinas, 1976].

The irradiance is defined as the radiation energy flux per unit area passing through an atmospheric layer and is sometimes referred to as the net flux. The irradiance is also composed of two parts: a direct solar beam and a diffuse flux. In a plane-parallel atmosphere the irradiance equivalents of (1)-(3) are

$$
\begin{gathered}
F_{0}(z, \lambda)=-\mu_{0} \pi F_{0} e^{-\tau(z) / \mu_{0}} \\
F_{D}(z, \lambda)=\int_{4 \pi} I_{\lambda}(z, \Omega) \mu d \Omega \\
F(z, \lambda)=F_{0}(z, \lambda)+F_{D}(z, \lambda),
\end{gathered}
$$

\footnotetext{
${ }^{1}$ Also at Earth and Space Sciences Division, Jet Propulsion Laboratory, California Institute of Technology, Pasadena.
}

Copyright 1992 by the American Geophysical Union. where $\mu$ is the cosine of the angle between $\Omega$ and the zenith. The irradiance is needed for calculations of radiative heating of the atmosphere.

Madronich [1987] considers the perturbation of the actinic flux inside and below optically thick clouds composed of conservative scatterers. He uses two approximate methods, two-stream and delta Eddington. The impact of clouds on the irradiance is examined by Shettle and Weinman [1970], who utilize the Eddington approximation. We will discuss a more accurate method for calculating the actinic flux and irradiance in a vertically inhomogeneous atmosphere with anisotropic, nonconservative scatterers. We will show that the introduction of an aerosol layer into a Rayleigh-scattering atmosphere can produce changes in the actinic flux and/or irradiance that are nonintuitive.

Consider a stratospheric $\mathrm{H}_{2} \mathrm{SO}_{4}$ aerosol layer formed by a volcanic eruption. This can be modeled as a nearly conservatively scattering layer in a Rayleigh-scattering atmosphere (layer B in Figure 1). Intuition suggests that the introduction of the scattering would lead to an increase in the actinic flux above the aerosol layer (region A in Figure 1) due to back scattering, or within the aerosol layer (region B) due to multiple scattering. However, we might not expect increases in the actinic flux beneath the aerosol layer (region C), for the following reasons. In region $\mathrm{C}$ there is additional actinic flux due to multiple scattering by the aerosol layer $B$, but we can argue that this flux is originally in the direct solar beam. It has been removed by layer $B$, which redistributes the radiation into the backward direction (region $\mathrm{A}$ ) and the forward direction (region C). Therefore it follows that the sum of the attenuated direct solar flux and the diffuse actinic flux in region $\mathrm{C}$ should not exceed the original actinic flux when the aerosol layer is absent. We might also anticipate that the absolute value of the irradiance would decrease at the surface with the introduction of the aerosol layer, reflecting the loss to space of backscattered photons. (In this paper, variation of the irradiance or its components will be discussed always with regard to absolute values.)

We will show, however, that under certain circumstances the actinic flux below the aerosol layer can increase without violating conservation of photons and under special conditions the irradiance also can increase below the aerosol layer, with respect to the clear sky situation. Our new radiative transfer model is described and utilized to explore the effects of aerosols.

\section{RADIATIVE TRANSFER MODEL}

Our radiative transfer model is a generalization of Gladstone's [1982] model for an inhomogeneous plane-parallel atmosphere 


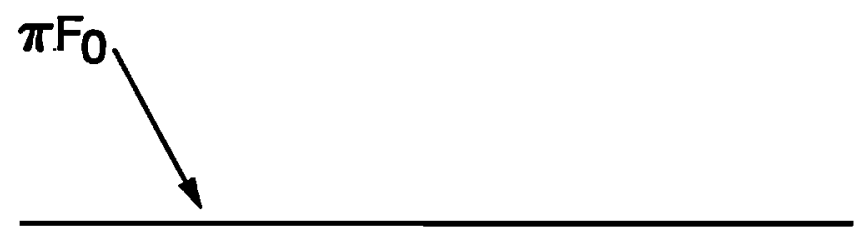

AEROSOL LAYER $\left(\tau_{1}, t, r\right)$

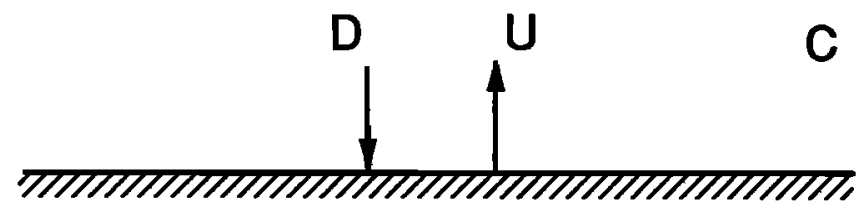

SURFACE $(\alpha)$

Fig. 1. Schematic of an atmosphere with a haze layer $(B)$ and clear layers $A$ and $C$, above and below (respectively) the haze layer. The total downwelling radiation $(D)$ is the sum of the direct solar beam $\left(\pi F_{0}\right)$ penetrating the aerosol layer of optical depth $\tau_{1}$ and the fraction of the upwelling radiation $U$ redirected downward by the aerosol layer (with transmissivity $t$ and reflectivity $r, t+r=1$ ). The upwelling radiation $U$ is a consequence of scattering off a surface with albedo $a$.

with isotropic scattering. The computational technique is based on the Feautrier method [Feautrier, 1964; Prather, 1976], applied to arbitrary anisotropic scattering.

The equation of radiative transfer for unpolarized radiation in a plane-parallel inhomogeneous atmosphere with anisotropic scattering is

$$
\begin{aligned}
\mu \frac{d I(\tau, \Omega)}{d \tau}= & I(\tau, \Omega)-\frac{\tilde{\omega}(\tau)}{4 \pi} \int_{4 \pi} P\left(\tau ; \Omega, \Omega^{\prime}\right) I\left(\tau, \Omega^{\prime}\right) d \Omega^{\prime} \\
& -\frac{F_{0}}{4} \tilde{\omega}(\tau) P\left(\tau ; \Omega,-\Omega_{0}\right) e^{-\tau / \mu_{0}},
\end{aligned}
$$

where $I$ is the specific intensity of diffuse radiation, $\pi F_{0}$ is the direct solar flux (photons $\mathrm{cm}^{-2} \mathrm{~s}^{-1}$ ), and all other symbols have their usual meanings [e.g., Liou, chap. 6, 1980]. For most phase functions of interest to the atmosphere we have $P\left(\tau ; \Omega, \Omega^{\prime}\right)=P\left(\tau ; \Omega \cdot \Omega^{\prime}\right)$ and we can expand the quantity $\widetilde{\omega}(\tau) P\left(\tau ; \Omega, \Omega^{\prime}\right)$ in a Fourier series in the azimuthal angle,

$$
\begin{aligned}
\tilde{\omega}(\tau) P\left(\tau ; \Omega, \Omega^{\prime}\right) & =\tilde{\omega}(\tau) P\left(\tau ; \Omega \cdot \Omega^{\prime}\right), \\
& =\sum_{l=0}^{N} \alpha_{l}(\tau) P_{l}\left(\Omega \cdot \Omega^{\prime}\right), \\
& =\sum_{m=0}^{N} \rho^{m}\left(\tau ; \mu, \mu^{\prime}\right) \cos m\left(\phi-\phi^{\prime}\right),
\end{aligned}
$$

where

$$
\rho^{m}\left(\tau ; \mu, \mu^{\prime}\right)=\sum_{l=m}^{N}\left(2-\delta_{0 m}\right) \alpha_{l}(\tau) \frac{(l-m) !}{(l+m) !} P_{l}^{m}(\mu) P_{l}^{m}\left(\mu^{\prime}\right) .
$$

Note that $P_{l}(\mu)$ and $P_{l}^{m}(\mu)$ are Legendre and associated Legendre polynomials, respectively, and $\delta_{0 m}$ is the Kronecker delta. The derivation of (5) follows standard procedures [Chandrasekhar, 1960; Liou, 1980]. In view of the expansion of the phase function we also may expand the intensity and the solar term in (4) in the form,

$$
\begin{aligned}
I(\tau ; \Omega) & =\sum_{m=0}^{N} I^{m}(\tau, \mu) \cos m\left(\phi-\phi_{0}\right) \\
\Sigma(\tau ; \Omega) & =\frac{F_{0}}{4} \tilde{\omega}(\tau) P\left(\tau ; \Omega,-\Omega_{0}\right) \\
& =\sum_{m=0}^{N} \Sigma^{m}(\tau ; \Omega) \cos m\left(\phi-\phi_{0}\right) .
\end{aligned}
$$

Upon inserting (5), (7), and (8) into (4), it can be shown that (4) splits up into $(N+1)$ independent equations,

$$
\begin{aligned}
& \mu \frac{d I^{m}(\tau, \mu)}{d \tau}=I^{m}(\tau, \mu)-\frac{1}{4}\left(1+\delta_{0 m}\right) \int_{-1}^{1} \rho^{m}\left(\tau ; \mu, \mu^{\prime}\right) I^{m}\left(\tau, \mu^{\prime}\right) d \mu^{\prime} \\
&-\Sigma^{m}(\tau, \mu) \\
&(m=0,1,2, \ldots, N) .(9)
\end{aligned}
$$

Since (9) is to be solved for each $m$, no confusion should arise by dropping the index $m$ in subsequent discussion, and we shall consider an equation of the type

$$
\mu \frac{d I(\tau, \mu)}{d \tau}=I(\tau, \mu)-\frac{1}{2} \gamma \int_{-1}^{1} \rho\left(\tau ; \mu, \mu^{\prime}\right) I\left(\tau, \mu^{\prime}\right) d \mu^{\prime}-\Sigma(\tau, \mu)
$$

where $\gamma=\left(1+\delta_{0 m}\right) / 2$.

Tosolve (10) numerically, we first approximate the angle variable $\mu(-1 \leq \mu \leq 1)$ by $2 n$ directions $\mu_{ \pm \mathrm{i}}(i=1,2, \ldots, n)$, where $\mu_{\mathrm{i}}>0$ and $\mu_{-i}=-\mu_{i}$. The usual choice for $\mu_{ \pm i}$ is the $2 n$ roots of the Legendre polynomial $\boldsymbol{P}_{2 n}(\boldsymbol{\mu})$. With this choice we can replace the integral over $\mu^{\prime}$ by the Gaussian quadrature formula and obtain a discretized version of (10),

$$
\begin{aligned}
& \mu_{i} \frac{d I\left(\tau, \mu_{j}\right)}{d \tau}=I\left(\tau, \mu_{i}\right)-\frac{1}{2} \gamma \sum_{j=1}^{n} a_{j}\left[\rho\left(\tau ; \mu_{i}, \mu_{j}\right) I\left(\tau, \mu_{j}\right)\right. \\
&\left.+\rho\left(\tau ; \mu_{i},-\mu_{j}\right) I\left(\tau,-\mu_{j}\right)\right]-\Sigma\left(\tau, \mu_{j}\right) \\
&-\mu_{i} \frac{d I\left(\tau,-\mu_{i}\right)}{d \tau}=I\left(\tau,-\mu_{i}\right)-\frac{1}{2} \gamma \sum_{j=1}^{n} a_{j}\left[\rho\left(\tau ;-\mu_{i}, \mu_{j}\right) I\left(\tau, \mu_{j}\right)\right. \\
&\left.+\rho\left(\tau ;-\mu_{i},-\mu_{j}\right) I\left(\tau,-\mu_{j}\right)\right]-\Sigma\left(\tau,-\mu_{i}\right)
\end{aligned}
$$

where $i=1,2,3, \ldots, n, a_{j}$ are the Gaussian weights, and $I\left(\tau, \mu_{i}\right)$ and $I\left(\tau,-\mu_{i}\right)$ represent upward and downward traveling streams of radiation, respectively. In order to achieve the high accuracy of the Gaussian quadrature formula the total number of Gaussian points, $2 n$, must exceed the number of terms in the expansion of the phase function (5). From the symmetry property of associated Legendre polynomials,

$$
P_{l}^{m}(-\mu)=(-1)^{l+m} P_{l}^{m}(\mu)
$$

we can derive two important symmetry properties of $\rho\left(\tau ; \mu, \mu^{\prime}\right)$ using (6),

$$
\begin{aligned}
& \rho\left(\tau ; \mu,-\mu^{\prime}\right)=\rho\left(\tau ;-\mu, \mu^{\prime}\right) \\
& \rho\left(\tau ;-\mu,-\mu^{\prime}\right)=\rho\left(\tau ; \mu, \mu^{\prime}\right) .
\end{aligned}
$$

Equations (11) and (12) can now be written compactly in vector and matrix notation,

$$
\begin{aligned}
& \frac{d u(\tau)}{d \tau}=A(\tau) v(\tau)-v_{0}(\tau) \\
& \frac{d v(\tau)}{d \tau}=B(\tau) u(\tau)-u_{0}(\tau),
\end{aligned}
$$

where 


$$
\begin{aligned}
u(\tau) & =\left(u_{1}(\tau), u_{2}(\tau), \ldots, u_{n}(\tau)\right), \\
v(\tau) & =\left(v_{1}(\tau), v_{2}(\tau), \ldots, v_{n}(\tau)\right), \\
u_{i}(\tau) & =\frac{1}{2}\left(I\left(\tau, \mu_{i}\right)+I\left(\tau,-\mu_{i}\right)\right), \\
v_{i}(\tau) & =\frac{1}{2}\left(I\left(\tau, \mu_{i}\right)-I\left(\tau,-\mu_{i}\right)\right), \\
u_{0}(\tau) & =\left(u_{01}(\tau), u_{02}(\tau), \ldots, u_{0 n}(\tau)\right), \\
v_{0}(\tau) & =\left(v_{01}(\tau), v_{02}(\tau), \ldots, v_{0 n}(\tau)\right), \\
u_{0 i}(\tau) & =\frac{1}{2 \mu_{i}}\left[\Sigma\left(\tau, \mu_{i}\right)+\Sigma\left(\tau,-\mu_{i}\right)\right], \\
v_{0 i}(\tau) & =\frac{1}{2 \mu_{i}}\left[\Sigma\left(\tau, \mu_{i}\right)-\Sigma\left(\tau,-\mu_{i}\right)\right],
\end{aligned}
$$

and $\mathrm{A}(\tau)$ and $\mathrm{B}(\tau)$ are $n \times n$ matrices, whose elements are given by

$$
\begin{aligned}
& A_{i j}(\tau)=\frac{1}{\mu_{i}}\left\{\delta_{i j}-\frac{1}{2} \gamma a_{j}\left[\rho\left(\tau ; \mu_{i}, \mu_{j}\right)-\rho\left(\tau ; \mu_{i},-\mu_{j}\right)\right]\right\} \\
& B_{i j}(\tau)=\frac{1}{\mu_{i}}\left\{\delta_{i j}-\frac{1}{2} \gamma a_{j}\left[\rho\left(\tau ; \mu_{i}, \mu_{j}\right)+\rho\left(\tau ; \mu_{i},-\mu_{j}\right)\right]\right\} .
\end{aligned}
$$

From (16) we have

$$
v(\tau)=A^{-1}\left\{\frac{d u(\tau)}{d \tau}+v_{0}(\tau)\right\}
$$

Substituting (18) into (17) yields

$$
\frac{d}{d \tau}\left\{A^{-1}(\tau) \frac{d u(\tau)}{d \tau}\right\}-B(\tau) u(\tau)=-\frac{d}{d \tau}\left(A^{-1}(\tau) v_{0}(\tau)\right)-u_{0}(\tau)
$$

Since $A(\tau)$ and $B(\tau)$ are positive definite matrices, (19) can be interpreted as the multicomponent (multiple stream) generalization of the steady state diffusion equation. Indeed, we can regard $u(\tau)$ as the "density of radiation," and $v(\tau)$ as the "flux of radiation" (not related to the flux defined in the introduction). Equation (18) states that the "flux" is proportional to the gradient of the "density." Equation (17) states that the "density" is proportional to the gradient of the "flux." This is what we expect for a system whose transport is described by diffusion.

Equation (19) is a generalization of Feautrier's equation of radiative transfer to anisotropic scattering and is therefore new. It has all the advantages, such as stability, of a steady state diffusion equation. The separation of the specific intensities into upward and downward traveling streams of radiation as described by (11)-(19) is similar to that in the "transfer matrix method" [Lenoble, 1985]. However, the latter is an exact analytic method which employs singular eigenfunctions and is applicable only to homogeneous layers. Our method is probably not different in principle from the method of discrete coordinates [Liou, 1975; Stamnes and Swanson, 1981]. However, in our case we do not need to assume that the solution must be a special form (eigenfunctions) and hence there is no need to compute any eigenvalues. As shown by Feautrier [1964], a convenient method for solving (19) is to discretize in $\tau$. The resulting equation may be cast into block tridiagonal form and can be easily and accurately inverted. The chief advantage of this method is the ability to deal with inhomogeneous atmospheres. Other advantages (not fully exploited in this work) include partial frequency redistribution (the nonmonochromatic problem, see Gladstone [1982]), generalization to two spatial coordinates [Mihalas et al., 1978], and incorporation of internal sources of radiation. The usefulness for these problems of the otherwise functionally equivalent (and widely used) "doubling and adding method" [Hansen and Travis, 1974] has not yet been demonstrated. The main restriction of the current method is that the atmospheric parameters must be piecewise continuous because of the occurrence of the term $A^{-1}(\tau)$ under the derivative sign in (19).
Equation (19) is solved numerically. Having solved for $\mathrm{u}(\tau)$, we obtain $\mathrm{v}(\tau)$ via (18), and from $\mathrm{u}(\tau)$ and $\mathrm{v}(\tau)$ we can derive $I\left(\tau, \pm \mu_{i}\right)$. The numerical calculations were checked for representative cases with the tables published by van de Hulst [1980]. These computations utilized the Henyey-Greenstein phase function [Henyey and Greenstein, 1941]:

$$
P_{H G}(\mu, g)=\frac{1-g^{2}}{\left(1+g^{2}-2 g \mu\right)^{3 / 2}}
$$

where the asymmetry parameter $g$ is defined as

$$
g=\int_{4 \pi} P_{H G}(\mu, g) \mu d \Omega \text {. }
$$

The details of our model comparison with the van de Hulst [1980] values are summarized in the appendix. These tests show that the radiative model is sufficiently accurate for practical atmospheric computations. We use Henyey-Greenstein's phase function for convenience. Our method is expected to work for the realistic Mie function. The only practical limitation is the amount of computer time.

For specific application to spherical planetary atmospheres a first-order correction can be implemented very simply. The direct beam attenuation factor, $e^{-\tau / \mu_{0}}$ in (4) is valid only in a plane-parallel atmosphere. This factor can be replaced with a function describing the attenuation in a spherical shell atmosphere [Froidevaux et al., 1985].

\section{IMPACT OF AN AEROSOL LAYER ON THE ACTINIC FLUX}

The perturbations of actinic fluxes by the introduction of an aerosol layer, such as the one resulting from the El Chichon volcanic eruption (see review by Hofmann [1987]), have been computed using our radiative model and are reported in Figures 1-3 of Michelangeli et al. [1989]. While, relative to a clear sky case, the enhancement of the actinic flux within the aerosol layer is expected, the enhancement below the aerosol layer is surprising (see Figure $3 b$ of Michelangeli et al [1989]).

It is easier to explain the cause of this enhancement in the context of a discussion of increases in the irradiance beneath an aerosol cloud, as we shall do in the next section. At this point, we state without proof that the increase in the actinic flux beneath a cloud is the result of photons being trapped between the cloud and the surface.

At least for one case, that of a layer of isotropic scatterers, the actinic flux can be computed directly from an exact integral formulation. The integral equation for the actinic flux in an atmosphere with isotropic scatterers overlying a Lambert surface with reflectivity $\alpha$ is [Yung and Goody, 1976, corrected herein]:

$$
\begin{gathered}
J(\tau, \lambda)=\left\{e^{-\tau / \mu_{0}}+2 \mu_{0} \alpha E_{2}\left(\tau_{1}-\tau\right) e^{-\tau_{1} / \mu_{0}}\right\} \pi F_{0}(\lambda) \\
+\widetilde{\omega}(\tau, \lambda) \int_{0}^{\tau_{1}}\left\{\frac{1}{2} E_{1}(|\tau-s|)+\alpha E_{2}\left(\tau_{1}-\tau\right) E_{2}\left(\tau_{1}-s\right)\right\} J(s, \lambda) d s,
\end{gathered}
$$

where we have used $\tau$ as the vertical variable instead of $z . E_{n}(x)$ is an exponential integral function of order $n$, and $\tau_{1}$ is the total optical depth. The first term on the right-hand side of $(20)$ is the direct solar flux term. The second term represents the reflected solar radiation. The first and second terms under the integral sign represent the scattering of diffuse radiation and reflected diffuse radiation, respectively. In this formalism the total diffuse actinic flux, $J_{D}(\tau, \lambda)$, is given by the sum of the second, third, and fourth term on the right-hand side of (20), and may be large enough to compensate for the attenuation of the direct flux. If (20) is solved using the single-scattering approximation, for the case of a conser- 
vative scatterer, $\alpha=0.25$, and $\mu_{0}=1$, there is a $5 \%$ increase in the actinic flux (relative to a clear sky) beneath a layer with $\tau_{1}=\mathbf{0 . 3 5}$.

For a surface reflectivity of 0.25 and conditions of overhead sun $\left(\mu_{0}=1\right)$ we use the full model (equation (19)) to explore the degree to which the actinic flux at the surface will increase as a function of the optical properties of the aerosol layer. There is no Rayleigh scattering in these calculations, because at visible wavelengths it is negligible compared with the El Chichon-enhanced aerosol scattering. For the range of values of single scattering albedo and phase function asymmetry parameter $(g)$ we identified the optical depth at which the increase in the actinic flux at the surface, relative to the clear sky case, was largest. Figures 2 and 3 show that the increase in the actinic flux at the surface will rise as the single scattering albedo of the aerosol increases. There is a slight correlation between the values for the phase function asymmetry parameter $(g)$ and scattering albedo at which the maximum increase in surface flux occurs. In addition, as the particle single scattering albedo increases, the optical depth corresponding to the maximum increase in surface actinic flux increases. It is clear that the enhancement is largest for highly forward scattering white aerosols in a layer of moderate optical depth $(\sim 1)$. The values reported in Figures 2 and 3 have a roughly linear correlation with surface albedo. The increase in actinic flux occurs primarily when the sun is near the zenith; the largest increase in actinic flux varies as $\mu_{0}^{n}$ where $n \geq 4$, while the optical depth at which the maximum increase occurs varies as $\mu_{0}^{3}$. The maximum increase in actinic flux in Figure 2 for an isotropic, conservative scatterer is $7 \%$ and the corresponding optical depth is $\mathbf{0 . 3 5}$; these values are in excellent agreement with the analytic result of (20). The sensitivity of these results to the choice of surface photometric function has not been explored for this paper.

It is interesting to note that (20) implies that the actinic flux will increase with decreasing altitude from the top of the atmosphere, through the aerosol layer, to the surface. Our model calculations do show this trend. However, in the absence of a source of radiation internal to the atmosphere, the irradiance to be discussed in the next section cannot increase with decreasing altitude (although the downward irradiance might) because it is subject to the constraints of energy conservation. Madronich [1987] discusses the change in actinic flux due to the presence of optically thick clouds composed of nonabsorbing, large particles, which are highly forward scattering $(g=0.875)$. This is more applicable to the consid-

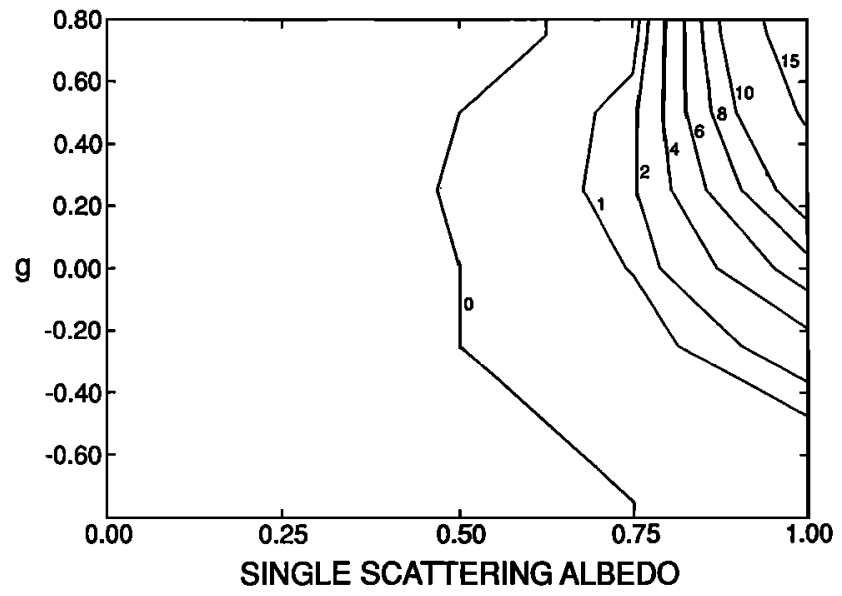

Fig. 2. The largest percentage increase in actinic flux at the surface, relative to a clear sky situation, as a function of aerosol properties, phase function asymmetry parameter $g$ and single scattering albedo $\tilde{\omega}$. These results are for $\mu_{0}=1$ and $\alpha=0.25$. erations of the impact of tropospheric water clouds. We repeated Madronich's calculation to compare numerical methods. For $\alpha=$ $0.05, \mu_{0}=\cos \left(20^{\circ}\right)$, and $\tau=8$, Madronich finds an increase in the actinic flux below the cloud (relative to the clear sky case; collimated solar beam incident on the cloud top) of $24 \%$ using a simple isotropic model and an increase of $14 \%$ using the more detailed delta-Eddington method. On the other hand, we calculate an increase of $5 \%$ with our more accurate method. We do not understand the differences between our result and the Madronich deltaEddington value. The delta-Eddington method has never been validated for use in actinic flux calculations, while our calculations compare well with the exact results tabulated in the appendix.

\section{IMPACT OF AN AEROSOL LAYER ON THE IRRADLANCE}

Shettle and Weinman [1970] have shown that the downward irradiance can increase to a level greater than the irradiance incident on the top of the atmosphere in the upper portion of an optically thick cloud of conservative scatterers. The clouds in their model were sufficiently optically thick for the downward irradiance below the clouds to be less than the incident solar irradiance. In this section we will consider the circumstances under which a stratospheric haze does lead to an increase in the surface irradiance, relative to the situation without the presence of the aerosol layer. For the case of a haze of conservative scatterers we present a simplified formulation paralleling the treatment in the appendix of Shettle and Weinman [1970], but being more explicit in the combination of parameters that would lead to a surface irradiance enhancement. Then utilizing our new numerical method, we will show that a surface enhancement in the irradiance may also result from the presence of a haze composed of nonconservative scatterers.

The downward and upward radiation streams projected onto the surface normal (i.e., irradiances), $D$ and $U$, respectively, are directly proportional to each other near the surface,

$$
U=-\alpha D
$$

where $\alpha$ is the albedo of the assumed Lambert surface (see Figure 1). The irradiance $S$ is also proportional to $D$,

$$
S=D+U=(1-\alpha) D \text {. }
$$

As such, any increase in $D$ means there is an equivalent increase in $S$, relative to the clear sky case. The following discussion is present-

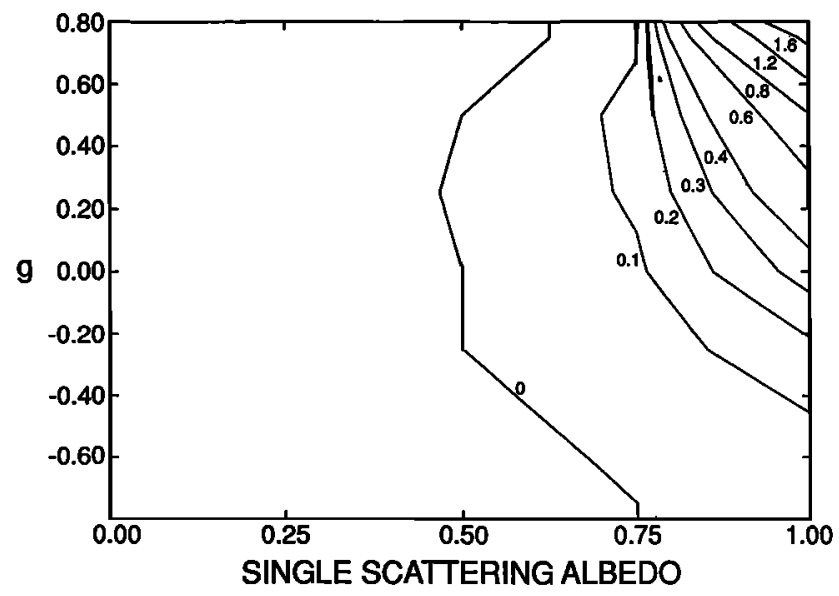

Fig. 3. The haze optical depth corresponding to the largest percentage increase in actinic flux displayed in Figure 2. 
ed in terms of changes in the downward irradiance for heuristic convenience.

Consider an aerosol layer of optical depth $\tau_{1}$ consisting of isotropic conservative scatterers overlying an optically thin atmosphere (see Figure 1). Sunlight can penetrate the layer with an attenuation factor given by $e^{-\tau / \mu_{0}}$. In the single scattering approximation, half of the scattered photons are directed into the forward beam. The upwelling radiation below the aerosol layer due to reflection by a Lambert surface is isotropic. Let the mean transmissivity and reflectivity of the aerosol layer with regard to this upwelling radiation be $t$ and $r$, respectively, such that

$$
t+r=1 \text {. }
$$

Adopting again the single scattering approximation,

$$
t=\frac{1}{2}\left[2 E_{3}\left(\tau_{1}\right)+1\right]
$$

where

$$
\begin{gathered}
E_{3}\left(\tau_{1}\right)=\int_{0}^{1} \mu e^{-\tau_{1} / \mu} d \mu \\
e^{-\tau_{1} / \mu}=2 E_{3}\left(\tau_{1}\right)
\end{gathered}
$$

and $\tau_{1} / \bar{\mu}$ defines the effective optical depth of the upwelling radiation. The second term in the parentheses on the right-hand side of (24) accounts for the photons scattered upward. From Figure 1 we have

and from (21),

$$
D=-\left(\frac{1}{2}\left(1+e^{-\tau_{1} / \mu_{0}}\right) \mu_{0} \pi F_{0}+r U\right)
$$

$$
D=\frac{-\frac{1}{2}\left(1+e^{-\tau_{1} / \mu_{0}}\right) \mu_{0} \pi F_{0}}{1-\alpha r}
$$

This implies a trapping ratio,

$$
\varepsilon=\frac{D}{-\mu_{0} \pi F_{0}}=\frac{\left(1+e^{-\tau_{1} / \mu_{0}}\right)}{2-\alpha\left[1-2 E_{3}\left(\tau_{1}\right)\right]}
$$

Note that for $\varepsilon>1$, we have $|D|>\mu_{0} \pi F_{0}$, i.e., $D$ exceeds the incident irradiance (which is the downward irradiance under clear sky conditions). When $\varepsilon>1$, it is easier for the photons to penetrate the aerosol layer than for the reflected photons to escape from this layer. The result is obviously a function of $\mu_{0}, \tau_{1}$, and $\alpha$. Values of $\mu_{0}$ and $\tau_{1}$ for which $\varepsilon=1$, as a function of $\alpha$, are illustrated in Figure 4. Since $S \propto D$, this figure also illustrates conditions for an increase in $S$ relative to the clear sky value. For a given value of $\alpha, D$ exceeds the unattenuated solar irradiance for larger values of $\mu_{0}$ and smaller values of optical depth. If the surface is highly reflective, $\varepsilon$ is greater than unity for a wide range of parameter values, but no enhancement is possible when $\alpha<$ 0.5 .

Shettle and Weinman [1970] point out that the increase in the downward irradiance relative to the value at the top of the atmosphere is a consequence of the trapping of photons between two reflective surfaces. The above derivation shows that, given a Lambertian surface photometric function, the ultimate cause of the "photon trapping" is the difference in the zenith angle of the incident solar beam and the effective zenith angle of the surfacereflected radiation. When $\mu_{0}>\bar{\mu}$, the optical path of the sunlight penetrating the cloud from above is smaller than the effective optical path for upwelling radiation entering the aerosol cloud from below. Consequently, the transmission through the cloud is larger (and reflection at the cloud surface is smaller) for the incident solar beam than for the reflected radiation. The upward curvature of the lines of constant $\alpha$ in Figure 4 reflect the nonlinear

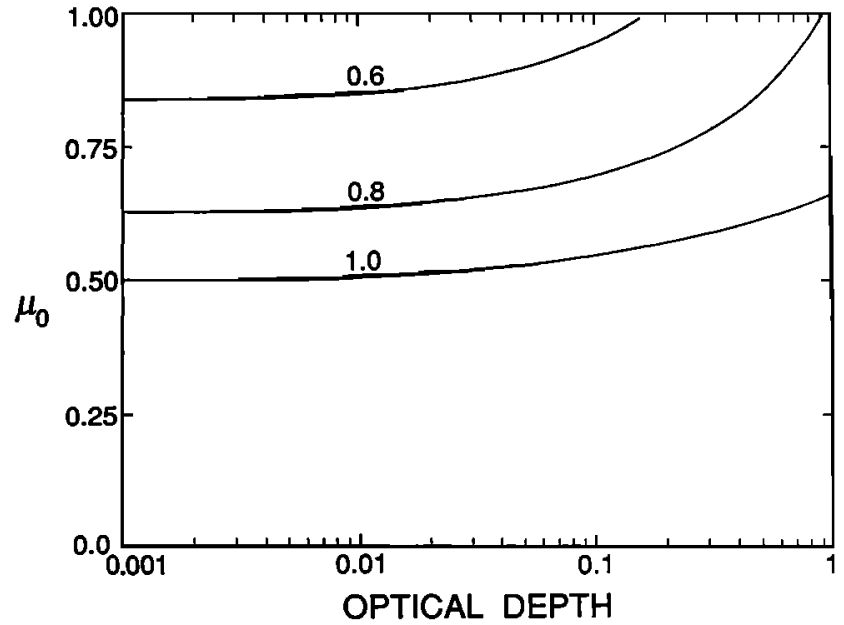

Fig. 4. Values of $\mu_{0}$ and optical depth for which $\varepsilon$ (irradiance trapping ratio) in the analytic expression (28) is unity. The value of $\alpha$ is varied and noted by each curve. For a given value of $\alpha, \varepsilon$ is greater than unity (downward irradiance at the surface is greater than unattenuated solar irradiance) in the region of the graph towards larger $\mu_{0}$ and smaller optical depth with reference to the $\varepsilon=1$ curve. There is no enhancement in surface irradiance for $\alpha \leq 0.5$.

increase in $\bar{\mu}$ with increasing cloud optical depth and the necessary increase in $\mu_{0}$ to obtain an enhanced surface irradiance. With a relatively reflective surface (large $\alpha$ ), the radiation is literally trapped between the cloud and the surface. As the surface becomes less reflective, the aerosol cloud needs to become a more effective "one-way mirror," so irradiation enhancements occur at a given cloud optical depth only at larger $\mu_{0}$.

Eliminating the simplifying assumptions of the previous discussion, we shall discuss the sensitivity of the increase in irradiance at the surface to the various optical parameters adopted in our numerical calculations for the presence of a haze layer. For a range of values for $\mu_{0}$ and $\alpha$ we identified the optical depth at which the increase in surface irradiance, relative to the clear sky situation, was largest. Figures 5 and 6 (no Rayleigh scattering included in computations) show the largest increases in surface irradiance and the corresponding haze layer optical depth as a function of $\mu_{0}$ and $\alpha$, key variables identified above. In these calculations, $\bar{\omega}=0.99$ and $g=0.7$ (typical values for the El Chichon aerosol). The irradiance at the surface increases by as much as a factor of 2 over a nearly white surface when the sun is close to the zenith, as expected from the heuristic discussion presented earlier. The largest changes in surface irradiance occur when the optical depth of the aerosol layer approaches unity. For $\alpha \geq 0.5$ and $\mu_{0} \geq 0.5$ there are correlations between $\mu_{0}$ and optical depth (at maximum irradiance increase), for a constant value of $\alpha$, and between $\alpha$ and optical depth, for a constantvalue of $\mu_{0}$, reminiscent of the curves in Figure 4. When $\widetilde{\omega}=0.99, \mu_{0}=1$, and $\alpha=0.85$, the maximum increase in surface irradiance is roughly independent of $g$, while the optical depth at which the maximum value occurs increases smoothly from $\tau=0.40$ for $g=-0.8$ to $\tau=1.5$ for $g=0.8$. On the other hand, for $\alpha=0.5$ a significant change in surface irradiance occurs only for highly forward scattering aerosol particles. Finally, the model produces a significant increase in irradiance only when $\tilde{\omega}$ approaches unity. However, we do find an increase of $\sim 1 \%$ for $\tilde{\omega}-0.92\left(g=0.7, \alpha=0.85, \mu_{0}=1\right)$ and even larger values as $\alpha$ approaches unity. We have therefore extended the results of Shetthe and Weinman [1970] with regard to increases in the downward irradiance over the solar value below clouds of conservative scatterers to include situations of clouds of nonconservative scatterers. 


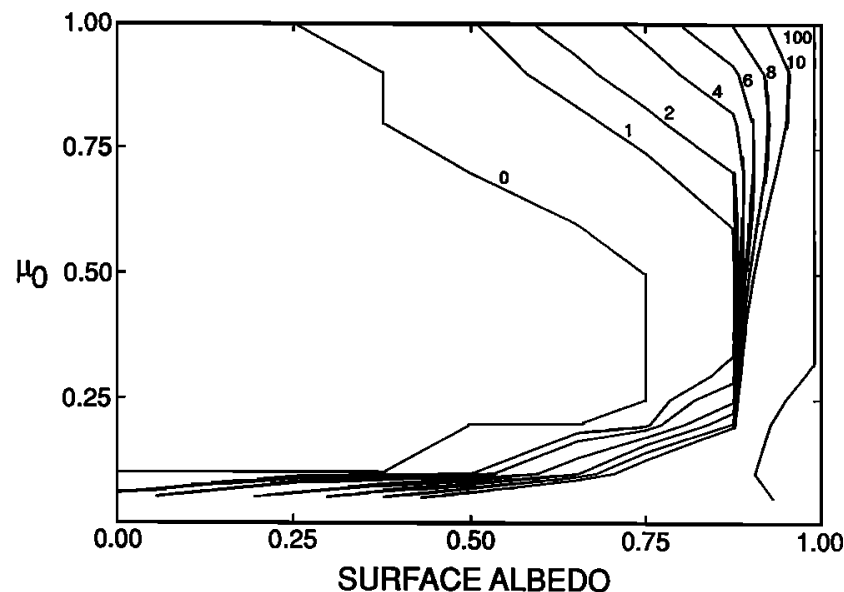

Fig. 5. The largest percentage increase in surface irradiance, relative to a clear sky situation, as a function of $\mu_{0}$ and $\alpha$. These results are for $\bar{\omega}=$ 0.99 and $g=0.7$, typical parameters for stratospheric volcanic aerosol particles.

In these calculations, both with and without dust, the irradiance $S$ is consistently directed downward from the top of the atmosphere to the surface. The absolute value of $S$ at the surface is always less than at the top of the atmosphere. This is an important consistency check on the numerical calculation that shows that energy is conserved.

\section{EFFECT OF EL CHICHON ERUPTION ON SURFACE UV-B FLUX}

In the summer of 1982 following the major spring eruption of the El Chichon volcano the optical depth of the resulting stratospheric aerosol cloud was -0.25 at $6000-7000 \AA$, as measured at $20^{\circ} \mathrm{N}$ latitude (Mauna Loa, Hawaii) [DeLuisi et al, 1983]. Values of $\tilde{\omega}=$ 0.99 and $g=0.7$ were characteristic of the El Chichon stratospheric haze particles; the particle extinction cross section was $\sim 1.4 \times 10^{-8}$ $\mathrm{cm}^{2}$ for wavelengths $\leq 2560 \AA$ increasing smoothly to $1.8 \times 10^{-8}$ at $8000 \AA$ [Pollack and Ackerman, 1983]. Inferring the vertical distribution of the stratospheric aerosols from observations, Michelangeli et al. [1989] report calculations of the change in actinic fluxes at $20^{\circ} \mathrm{N}$ in the summer of 1982 due to the presence of the volcanic aerosol layer but with no changes in species profiles, particularly $\mathrm{O}_{2}$ and $\mathrm{O}_{3}$. In these computations, Rayleigh scattering and gas opacity were included. A globally averaged surface albedo of 0.25 [Goody and Yung, 1989] was adopted.

The Michelangeli et al. [1989] results for changes (relative to the clear sky case) in the surface actinic fluxes in the UV-B spectral range at a local time of 1400 (solar zenith angle $\chi=45^{\circ}$ ) are presented in Table 1 . The changes in the surface irradiances generated in the same set of calculations are very close to the values reported in Table 1. Of particular note is the increase in the surface actinic flux at the short wavelength end $(\lambda<3000 \AA)$ of the UV-B spectral range, by as much as $45 \%$ at $2900 \AA$. The magnitude of this increase in actinic flux is much larger than the increases in actinic flux displayed in Figure 2. In the latter case, we made a comparison between a "clear" sky with negligible gas optical depth and a sky with a significant aerosol layer. On the other hand, in Table 1 we report again a comparison between situations with and without dust, but in both cases the optical depth for gas absorption increases to large values with decreasing wavelength below $-3000 \AA$. The introduction of a layer of nearly white, forward scattering particles at about the altitude of the peak gas absorption enhances the penetration of photons to the clearer

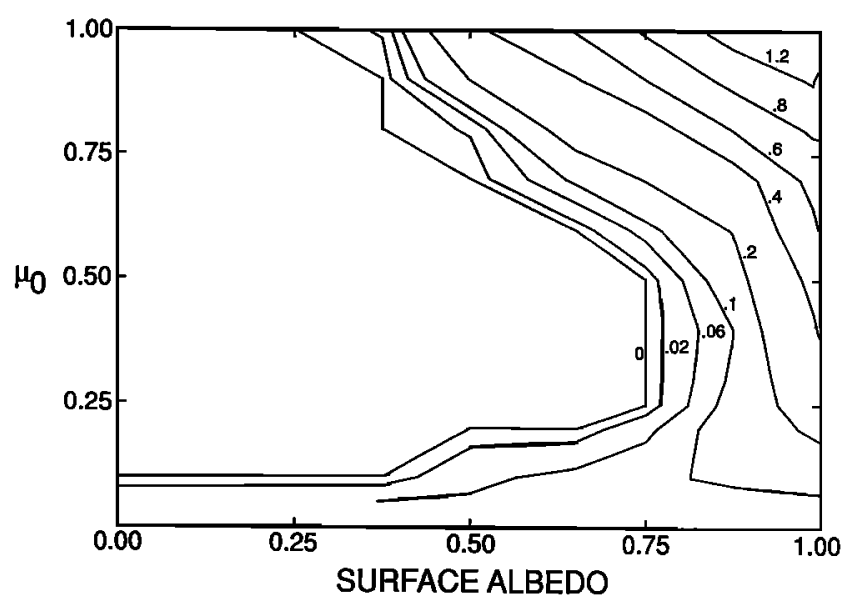

Fig. 6. The haze optical depth corresponding to the largest percentage increase in irradiance displayed in Figure 5.

atmosphere below, which would otherwise be nearly devoid of radiation at these wavelengths with large gas absorption optical depths.

The short wavelength UV-B radiation that does penetrate to the Earth's surface is particularly harmful to humans and other living species [National Academy of Sciences, 1982]. The calculated increase in the radiation field occurs without any change in the overhead ozone column. Additional perturbations to stratospheric chemistry that result from the introduction of volcanic aerosols, such as a decrease in stratospheric $\mathrm{O}_{3}$ [see Michelangeli et al, 1989; Hofmann and Solomon, 1989], may cause a further increase in the surface fluxes at UV-B wavelengths.

\section{SUMMARY}

An accurate radiative transfer model for inhomogeneous atmospheres with anisotropic scattering has been introduced and used to investigate the effect of an aerosol layer on two important radiometric quantities. Circumstances in which there are enhancements, relative to the clear sky cases, in the atmospheric radiation beneath the aerosol layer are investigated in detail. Heuristic arguments suggest that these effects are the result of "photon trapping" as a consequence of the multiple scattering. Calculations with the new radiative model show that an aerosol layer with optical depth $\sim 1$, composed of highly reflective, forward scattering particles, will result in an increase in the actinic flux at the surface of as much as $15 \%$ for small zenith angles and a surface reflectivity of 0.25. An even larger increase occurs over surfaces with larger albedos. The presence of such a haze results in a $100 \%$ increase in the surface irradiance over a nearly white surface when the sun is overhead.

TABLE 1. Variation in Surface Actinic Flux due to Stratospheric Volcanic Aerosols

\begin{tabular}{cc}
\hline Wavelength, $\AA$ & Change, $\%$ \\
\hline 2900 & +45 \\
2950 & +3 \\
3000 & -5 \\
3050 & -11 \\
3100 & -8 \\
3150 & -7 \\
3200 & -6 \\
\hline
\end{tabular}

At $20^{\circ} \mathrm{N}$ latitude, summer, $1400 \mathrm{LT}$ (solar zenith angle $\chi=45^{\circ}$ ) 
These results have obvious implications for the photochemistry, and possibly the thermal structure, of the troposphere after a volcanic eruption, or other geophysical phenomena resulting in a haze of bright, forward scattering particles. In particular, the eruption of El Chichon produced a stratospheric aerosol layer of optical depth $\sim 0.25$, consisting of particles with $\widetilde{\omega}=0.99$ and $g=0.7$. One calculated consequence is a doubling in the surface irradiance at $2900 \AA$, in the UV-B spectral range, a wavelength particularly harmful to humans and other elements of our ecosystem. This is a result of simply introducing the stratospheric aerosol haze; further perturbations to the chemistry of the stratosphere, in particular a reduction in $\mathrm{O}_{3}$ abundances, may lead to additional increases in the UV surface fluxes.

\section{APPENDIX: CALCULATION OF REFLECTION}

\section{AND TRANSMISSION FOR SAMPLE CASES}

The algorithm used in our radiative transfer model is reviewed in the text in detail. The model is designed for calculations of radiative transfer of unpolarized radiation in a plane-parallel inhomogeneous atmosphere with anisotropic scattering. Because of the complexity of the problem to be solved, it is difficult to find a method to check the accuracy of the numerical results comprehensively. Instead, we choose several simple but representative cases and compare our numerical results with the results listed in the work by van de Hulst [1980]. Using a totally different algorithm (doubling method), the latter contains the most detailed compilation we could find of radiative transfer calculations in a homogeneous plane-parallel atmosphere with anisotropic scattering. Shown in Table A1 are values (as calculated by the present algorithm) for the reflection $R\left(\mu_{0}, \mu\right)$ from the top of, and transmission $T\left(\mu_{0}, \mu\right)$ through, a finite layer. The layer is defined by a total optical depth $b$ of scatterers with single scattering albedo $a$ and phase function asymmetry parameter $g$. The illumination and viewing geometry are specified by $\mu_{0}$, the cosine of the incident angle, and $\mu$, the cosine of the observation angle. At $\mu=\mu_{0}, T\left(\mu_{0}, \mu\right)$ includes the direct transmitted beam. The accuracy of our results depends on the choice of the optical depth grid at which the calculations are performed. For the calculations reported in Table A1 the minimum nonzero optical depth in the computations was $10^{-3}$. Between $b=10^{-3}$ and $10^{-1}$, the grid was divided into 10 points per decade of optical depth values. Between $b=10^{-1}$ and 2 (or the maximum optical depth for the calculation, whichever was smaller), the grid points were incremented by a constant value, $\leq 0.01$. If $b$ was greater than 2 , the remaining range in optical depth was divided into more than 10 points per decade of optical depth range. The accuracy of our results also depends on the appropriate choice of number of terms in the spherical harmonic expansion. For $g=0$ and 0.5 the number of spherical harmonic terms was 40,

TABLE A1. Comparison of Results for $R\left(\mu_{0}, \mu\right)$ and $T\left(\mu_{0}, \mu\right)$

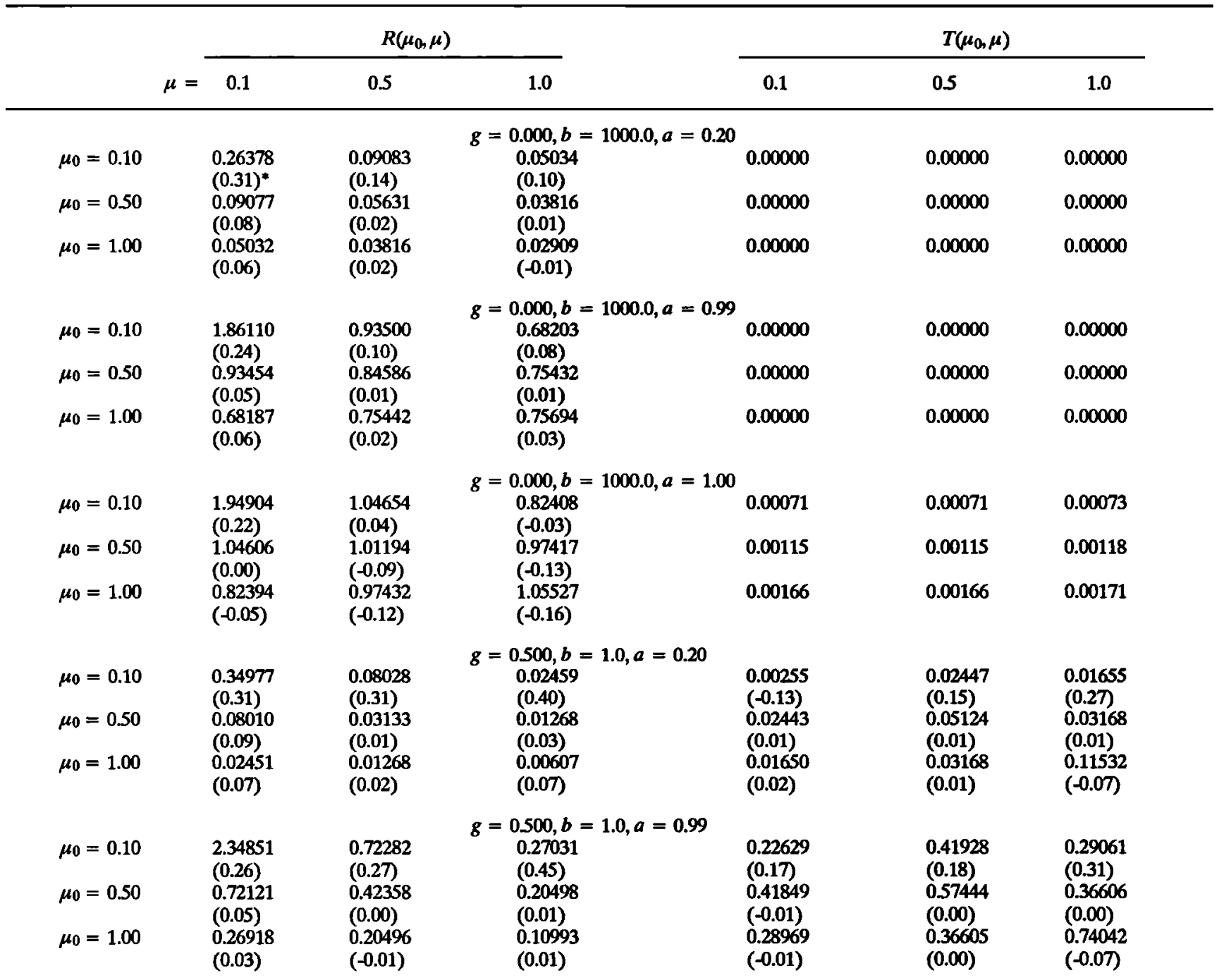


TABLE A1. (continued)

\begin{tabular}{|c|c|c|c|c|c|c|}
\hline & \multicolumn{3}{|c|}{$R\left(\mu_{0}, \mu\right)$} & \multicolumn{3}{|c|}{$T\left(\mu_{0}, \mu\right)$} \\
\hline & $\mu=$ & 0.5 & 1.0 & 0.1 & 0.5 & 1.0 \\
\hline $\begin{array}{l}\mu_{0}=0.10 \\
\mu_{0}=0.50 \\
\mu_{0}=1.00\end{array}$ & $\begin{array}{l}2.38609 \\
(0.26) \\
0.73748 \\
(0.05) \\
0.27640 \\
(0.03)\end{array}$ & $\begin{array}{l}0.73913 \\
(0.27) \\
0.43579 \\
(0.00) \\
0.21141 \\
(0.00)\end{array}$ & $\begin{array}{c}g=0.500, b=1.0, a=1.00 \\
0.27756 \\
(0.45) \\
0.21144 \\
(0.01) \\
0.11356 \\
(0.00)\end{array}$ & $\begin{array}{l}0.23624 \\
(0.17) \\
0.43159 \\
(-0.01) \\
0.29843 \\
(-0.01)\end{array}$ & $\begin{array}{l}0.43241 \\
(0.18) \\
0.58912 \\
(0.00) \\
0.37525 \\
(0.00)\end{array}$ & $\begin{array}{l}0.29938 \\
(0.31) \\
0.37526 \\
(0.01) \\
0.75174 \\
(-0.07)\end{array}$ \\
\hline $\begin{array}{l}\mu_{0}=0.10 \\
\mu_{0}=0.50 \\
\mu_{0}=1.00\end{array}$ & $\begin{array}{l}0.34988 \\
(0.34) \\
0.08020 \\
(0.10) \\
0.02461 \\
(0.09)\end{array}$ & $\begin{array}{l}0.08045 \\
(0.41) \\
0.03229 \\
(0.04) \\
0.01375 \\
(0.00)\end{array}$ & $\begin{aligned} & g= 0.500, b=4.0, a=0.20 \\
& 0.02473 \\
&(0.58) \\
& 0.01375 \\
&(0.03) \\
& 0.00752 \\
&(0.11)\end{aligned}$ & $\begin{array}{l}0.00003 \\
(14.33) \\
0.00018 \\
(-0.50) \\
0.00119 \\
(0.57)\end{array}$ & $\begin{array}{l}0.00018 \\
(-0.44) \\
0.00089 \\
(-0.02) \\
0.00343 \\
(0.09)\end{array}$ & $\begin{array}{l}0.00119 \\
(0.80) \\
0.00343 \\
(0.10) \\
0.02552 \\
(-0.07)\end{array}$ \\
\hline $\begin{array}{l}\mu_{0}=0.10 \\
\mu_{0}=0.50 \\
\mu_{0}=1.00\end{array}$ & $\begin{array}{l}2.43059 \\
(0.30) \\
0.86662 \\
(0.05) \\
0.42958 \\
(0.03)\end{array}$ & $\begin{array}{l}0.86941 \\
(0.38) \\
0.68507 \\
(0.01) \\
0.49165 \\
(0.00)\end{array}$ & $\begin{array}{c}g=0.500, b=4.0, a=0.99 \\
0.43180 \\
(0.55) \\
0.49173 \\
(0.02) \\
0.43629 \\
(0.01)\end{array}$ & $\begin{array}{l}0.09232 \\
(0.46) \\
0.15724 \\
(0.12) \\
0.22312 \\
(0.11)\end{array}$ & $\begin{array}{l}0.15761 \\
(0.35) \\
0.26934 \\
(0.01) \\
0.37193 \\
(0.00)\end{array}$ & $\begin{array}{l}0.22369 \\
(0.37) \\
0.37197 \\
(0.01) \\
0.59127 \\
(-0.03)\end{array}$ \\
\hline $\begin{array}{l}\mu_{0}=0.10 \\
\mu_{0}=0.50 \\
\mu_{0}=1.00\end{array}$ & $\begin{array}{l}2.47991 \\
(0.30) \\
0.90181 \\
(0.05) \\
0.45736 \\
(0.03)\end{array}$ & $\begin{array}{l}0.90472 \\
(0.38) \\
0.72764 \\
(0.02) \\
0.53093 \\
(0.00)\end{array}$ & $\begin{array}{cl}g= & 0.500, b=4.0, a=1.00 \\
& 0.45968 \\
& (0.54) \\
0.53100 \\
(0.02) \\
0.47598 \\
(0.01)\end{array}$ & $\begin{array}{l}0.10481 \\
(0.46) \\
0.17695 \\
(0.11) \\
0.24673 \\
(0.11)\end{array}$ & $\begin{array}{l}0.17737 \\
(0.35) \\
0.30035 \\
(0.00) \\
0.40838 \\
(0.00)\end{array}$ & $\begin{array}{l}0.24736 \\
(0.37) \\
0.40842 \\
(0.01) \\
0.63414 \\
(-0.03)\end{array}$ \\
\hline $\begin{array}{l}\mu_{0}=0.10 \\
\mu_{0}=0.50 \\
\mu_{0}=1.00\end{array}$ & $\begin{array}{l}2.51841 \\
(0.30) \\
0.96653 \\
(0.05) \\
0.55041 \\
(0.03)\end{array}$ & $\begin{array}{l}0.96964 \\
(0.37) \\
0.83678 \\
(0.01) \\
0.68785 \\
(0.01)\end{array}$ & $\begin{aligned} g= & 0.500, b=8.0, a=1.00 \\
& 0.55302 \\
& (0.51) \\
& 0.68792 \\
& (0.02) \\
& 0.70161 \\
& (0.01)\end{aligned}$ & $\begin{array}{l}0.06597 \\
(0.80) \\
0.11075 \\
(0.45) \\
0.16105 \\
(0.44)\end{array}$ & $\begin{array}{l}0.11060 \\
(0.31) \\
0.18569 \\
(-0.03) \\
0.26984 \\
(-0.04)\end{array}$ & $\begin{array}{l}0.16089 \\
(0.34) \\
0.26994 \\
(0.00) \\
0.39449 \\
(0.00)\end{array}$ \\
\hline $\begin{array}{l}\mu_{0}=0.10 \\
\mu_{0}=0.50 \\
\mu_{0}=1.00\end{array}$ & $\begin{array}{l}256365 \\
(0.31) \\
1.04251 \\
(0.06) \\
0.66106 \\
(0.05)\end{array}$ & $\begin{array}{l}1.04583 \\
(0.37) \\
0.96474 \\
(0.02) \\
0.87419 \\
(0.02)\end{array}$ & $\begin{array}{c}g=0.500, b=32.0, a=1.00 \\
0.66393 \\
(0.48) \\
0.87418 \\
(0.02) \\
0.97288 \\
(0.04)\end{array}$ & $\begin{array}{l}0.02725 \\
(33.76) \\
0.04574 \\
(33.28) \\
0.06660 \\
(33.31)\end{array}$ & $\begin{array}{l}0.03411 \\
(-0.61) \\
0.05727 \\
(-0.94) \\
0.08338 \\
(-0.92)\end{array}$ & $\begin{array}{l}0.04986 \\
(-0.21) \\
0.08370 \\
(-0.54) \\
0.12188 \\
(-0.52)\end{array}$ \\
\hline $\begin{array}{l}\mu_{0}=0.10 \\
\mu_{0}=0.50 \\
\mu_{0}=1.00\end{array}$ & $\begin{array}{l}3.64024 \\
(0.08) \\
0.52976 \\
(0.01) \\
0.11370 \\
(0.01)\end{array}$ & $\begin{array}{l}0.53043 \\
(0.14) \\
0.16641 \\
(0.01) \\
0.04618 \\
(0.00)\end{array}$ & $\begin{aligned} g= & 0.750, b=0.5, a=1.00 \\
& 0.11415 \\
& (0.40) \\
& 0.04618 \\
& (0.01) \\
& 0.01532 \\
& (0.07)\end{aligned}$ & $\begin{array}{l}0.99148 \\
(0.03) \\
0.66607 \\
(0.00) \\
0.15443 \\
(0.00)\end{array}$ & $\begin{array}{l}0.66657 \\
(0.08) \\
0.83852 \\
(0.01) \\
0.14574 \\
(0.00)\end{array}$ & $\begin{array}{l}0.15487 \\
(0.29) \\
0.14574 \\
(0.00) \\
2.30035 \\
(-0.16)\end{array}$ \\
\hline $\begin{array}{l}\mu_{0}=0.10 \\
\mu_{0}=0.50 \\
\mu_{0}=1.00\end{array}$ & $\begin{array}{l}0.48446 \\
(0.39) \\
0.05062 \\
(0.11) \\
0.01035 \\
(0.03)\end{array}$ & $\begin{array}{l}0.05085 \\
(0.58) \\
0.01481 \\
(0.12) \\
0.00494 \\
(0.10)\end{array}$ & $\begin{aligned} g= & 0.750, b=1.0, a=0.20 \\
& 0.01040 \\
& (0.50) \\
& 0.00494 \\
& (0.12) \\
& 0.00218 \\
& (0.39)\end{aligned}$ & $\begin{array}{l}0.00293 \\
(0.13) \\
0.02236 \\
(-0.01) \\
0.00784 \\
(0.02)\end{array}$ & $\begin{array}{l}0.02241 \\
(0.20) \\
0.09319 \\
(0.02) \\
0.01779 \\
(0.03)\end{array}$ & $\begin{array}{l}0.00787 \\
(0.35) \\
0.01779 \\
(0.03) \\
0.53026 \\
(-0.16)\end{array}$ \\
\hline $\begin{array}{l}\mu_{0}=0.10 \\
\mu_{0}=0.50 \\
\mu_{0}=1.00\end{array}$ & $\begin{array}{l}3.65798 \\
(0.35) \\
0.61301 \\
(0.05) \\
0.14660 \\
(0.03)\end{array}$ & $\begin{array}{l}0.61684 \\
(0.67) \\
0.29666 \\
(0.03) \\
0.09764 \\
(0.01)\end{array}$ & $\begin{aligned} g= & 0.750, b=1.0 a=0.99 \\
& 0.14905 \\
& (1.77) \\
& 0.09768 \\
& (0.05) \\
& 0.03786 \\
& (0.15)\end{aligned}$ & $\begin{array}{l}0.36066 \\
(0.35) \\
0.56448 \\
(0.00) \\
0.20646 \\
(0.00)\end{array}$ & $\begin{array}{l}0.56684 \\
(0.42) \\
0.93553 \\
(0.01) \\
0.25960 \\
(0.00)\end{array}$ & $\begin{array}{l}0.20872 \\
(1.09) \\
0.25964 \\
(0.01) \\
3.02341 \\
(-0.16)\end{array}$ \\
\hline
\end{tabular}


TABLE A1. (continued)

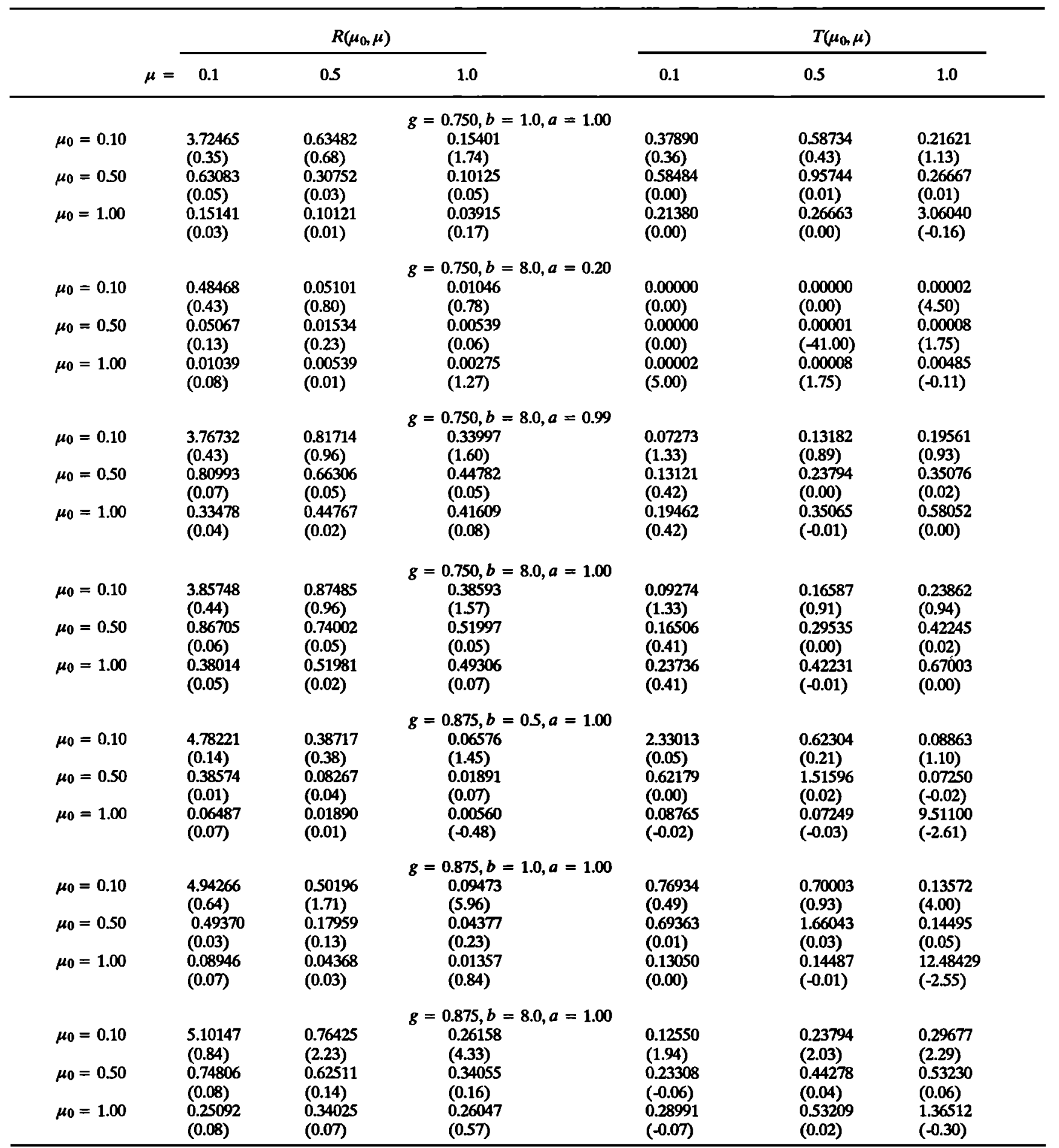

\footnotetext{
*Values in parentheses are the percentage differences between results calculated with the methods described in this paper and those reported
} by van de Hulst [1980].

and for $g=0.75$ and $0.875,80$. The number of Gaussian angles in the calculations was always equal to half of the number of spherical harmonic terms. Our model results have been azimuthally averaged and normalized by $\mu_{0}$ to enable a comparison with the van de Hulst [1980] values. The differences between our values and those reported by van de Hulst [1980] were typically less than $1 \%$. Table A1 shows where the parameter range covered by van de Hulst [1980] our results differed by more than $1 \%$ (the percentage differences between our results and those of van de Hulst are the numbers enclosed in parentheses). In all of these calculations, the total number of grid points is 320 . To compare with the van de Hulst [1980] results for $b=\infty$, we carried out runs with $b=1000$. Some of the large differences were the consequence of only one significant digit being printed by van de Hulst [1980]. For large forward scattering, $g=0.875$, we found discrepancies when $\mu_{0}$ or $\mu$ were 0.1 , but the discrepancies could be reduced by decreasing spacing in the optical depth grid or increasing the number of spherical harmonic terms. The largest discrepancy shown in Table A1, $-33 \%$ at $g=0.5$ and $b=32$ (the largest optical depth in a nonisotropic case that is calculated by van de Hulst [1980], was 
easily reduced to $1-2 \%$ by an increase in the density of optical depth grid points between 2 and 32 . In summary, we feel our model overall is sufficiently accurate for practical atmospheric calculations.

Acknowledgment. We thank J. Pollack and the anonymous referees for their extremely helpful comments. This research is supported by EPA grant 9D4125NALXX to the California Institute of Technology. Contribution number 4685 from the Division of Geological and Planetary Sciences, California Institute of Technology, Pasadena, California 91125.

\section{REFERENCES}

Chandrasekhar, S., Radiative Transfer, Dover, New York, 1960.

DeLuisi, J. J., E. G. Dutton, K. L. Coulson, T. E. DeFoor, and B. G. Mendonca, On some radiative features of the $\mathrm{El}$ Chichon volcanic stratospheric dust cloud and a cloud of unknown origin observed at Mauna Loa, J. Geophys. Res., 88, 6769-6772, 1983.

Feautrier, P., Sur la résolution numérique de l'équation de transfert, $C$. $R$ Acad Sci Paris, 258, 3189-3194, 1964.

Froidevaux, L., M. Allen, and Y. L. Yung, A critical analysis of $\mathrm{ClO}$ and $\mathrm{O} 3$ in the mid-latitude stratosphere, J. Geophys. Res. , 90, 12,999-13,029, 1985.

Gladstone, G. R., Radiative transfer with partial frequency redistribution in inhomogeneous atmospheres: Application to the Jovian aurora, $J$. Quant Spectrosc. Radiat Transfer, 27, 545-556, 1982.

Goody, R. M., and Y. L. Yung, Atmospheric Radiation: Theoretical Basis, 2nd ed., Oxford University Press, New York, 1989.

Hansen, J. E. and L. D. Travis, Light scattering in planetary atmospheres, Space Sci. Rev., 16, 527-610, 1974.

Henyey, L. C., and J. L. Greenstein, Diffuse radiation in the galaxy, Astrophys. J., 93, 70-83, 1941.

Hofmann, D. J., Perturbations to the global atmosphere associated with the El Chichon volcanic eruption of 1982, Rev. Geophys., 25, 743-759, 1987.

Hofmann, D. J., and S. Solomon, Ozone destruction through heterogeneous chemistry following the eruption of El Chichón, $J$. Geophys. Res., 94, 5029-5041, 1989.

Lenoble, J. (ed.), Radiative Transfer in Scattering and Absorbing Atmospheres: Standard Computational Procedures, A. Deepak, Hampton, V.A., 1985.

Liou, K N., Applications of the discrete-ordinate method for radiative transfer to inhomogeneous aerosol atmospheres, J. Geophys. Res., 80, 3434-3440, 1975.

Liou, K-N., An Introduction to Atmospheric Rodiation, San Diego, Calif., 1980.

Luther, F. M., and R. J. Gelinas, Effect of molecular multiple scattering and surface albedo on atmospheric photodissociation rates, J. Geophys Res., 81, 1125-1132, 1976.

Madronich, S., Photodissociation in the atmosphere 1. Actinic flux and the effects of close-up ground reflections and clouds, J. Geophys. Res., 92, 9740-9752, 1987.

Michelangeli, D., M. Allen, and Y. L. Yung, The effect of El Chichon volcanic aerosols on the chemistry of the stratosphere through radiative coupling, J. Geophys. Res. 94, 18,429-18,443, 1989.

Mihalas, D., L. H. Auer, and B. R. Mihalas, Two-dimensional radiative transfer, I, Planar geometry, Astrophys. J., 220, 1001-1023, 1978.

National Academy of Sciences, Causes and Effects of Stratospheric Ozone Reduction: An Update, National Academy Press, Washington, D.C., 1982.

Pollack, J. B., and T. P. Ackerman, Possible effects of the El Chichon volcanic cloud on the radiation budget of the northem tropics, Geophys. Res. Lett, 10, 1057-1060, 1983.

Prather, M. J., Solution of the inhomogeneous Rayleigh scattering atmosphere, Astrophys. J., 192, 787-792, 1976.

Shettle, E. P., and J. A. Weinman, The transfer of solar irradiance through inhomogeneous turbid atmospheres evaluated by Eddington's approximation, J. Atmos. Sci., 27, 1048-1055, 1970.

Stamnes, K., and R. A. Swanson, A new look at the discrete ordinate method for radiative transfer calculations in anisotropically scattering atmosphere, J. Atmos. Sci, 38, 387-399, 1981.

van de Hulst, H. C., Multiple Light Scattering, vols. I and II, Academic, San Diego, Calif., 1980

Wofsy, S. C., Temporal and latitudinal variations of stratospheric trace gases: A critical comparison between theory and experiment,J. Geophys. Res. 83, 364-378, 1978.

Yung, Y. L, A numerical method for calculating the mean intensity in an inhomogeneous Rayleigh scattering atmosphere, J. Quank. Spectrasc. Radiat. Transfer, 16, 755-761, 1976.

Yung, Y. L., and R. M. Goody, Photometric properties of the surface of Io and their influence on line formation in the atmosphere, Icarus, 29, $57-67,1976$

M. Allen and D. Crisp, Earth and Space Sciences Division, Jet Propulsion Laboratory, California Institute of Technology, $\mathbf{4 8 0 0}$ Oak Grove Drive, Pasadena, CA 91109.

J. Eluszkiewicz, D. V. Michelangeli, R.L. Shia, and Y. L. Yung, Division of Geological and Planetary Sciences, California Institute of Technology, Pasadena, CA 91125.

(Received November 28, 1989; revised May 8, 1991; accepted May 8, 1991.) 